\title{
Estudo prospectivo das lesões musculares em três temporadas consecutivas do Campeonato Brasileiro de Futebol* $^{*}$
}

\section{Prospective Study of Muscle Injuries in Three Consecutive Seasons of the Brazilian Football Championship}

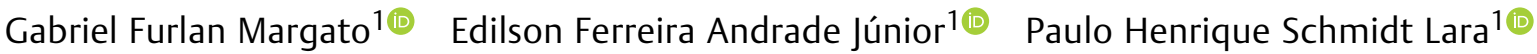 \\ Jorge Roberto Pagura ${ }^{20}$ Moisés Cohen ${ }^{30}$ Gustavo Gonçalves Arliani ${ }^{10}$
}

\footnotetext{
${ }^{1}$ Centro de Traumatologia do Esporte, Escola Paulista de Medicina, Universidade Federal de São Paulo, São Paulo, São Paulo, Brasil

2 Departamento de Neurologia e Neurocirurgia, Faculdade de Medicina do ABC, Santo André, SP, Brasil

${ }^{3}$ Departamento de Ortopedia e Traumatologia, Escola Paulista de Medicina, Universidade Federal de São Paulo, São Paulo, Brasil
}

\author{
Endereço para correspondência Paulo Henrique Schmidt Lara, MD, \\ Centro de Traumatologia do Esporte, Escola Paulista de Medicina, \\ Universidade Federal de São Paulo, Rua Estado de Israel, 636, Vila \\ Clementino, São Paulo, SP, 04022-001, Brasil \\ (e-mail: phslara@gmail.com).
}

Rev Bras Ortop 2020;55(6):687-694.

\begin{abstract}
Resumo
Palavras-chave

- traumatismos em atletas

- atletas

- futebol

- músculos isquiotibiais

Objetivo Realizar uma avaliação prospectiva das lesões musculares ocorridas durante as partidas das séries A e B do Campeonato Brasileiro de Futebol Masculino dos anos de 2016 a 2018.

Métodos Estudo de coorte prospectivo com coleta de dados referentes às lesões musculares ocorridas durante os jogos oficiais da primeira e segunda divisões do Campeonato Brasileiro de Futebol Masculino nas temporadas de 2016, 2017 e 2018. Resultados O número total de lesões musculares foi de 577 ao longo das 3 temporadas, havendo uma redução gradual e anual na incidência delas (219 lesões em 2016, 195 em 2017, e 163 em 2018), com diferença estatística significativa entre os anos de 2016 e 2018. As lesões musculares representaram aproximadamente $35 \%$ de todas as lesões. A incidência das lesões musculares foi 7,66 para cada 1.000 horas de jogo. Nas 3 temporadas (2016 a 2018), a mais comum foi a lesão muscular dos isquiotibiais $(41,1 \%, 40,5 \%$ e 33,7\%, respectivamente). Os laterais foram os mais acometidos, e a escala de severidade de lesão mais comum foi a moderada ( 8 a 28 dias). O momento da partida com maior incidência de lesões foi no período entre 61 e 75 minutos, com um índice de 19,9\%, não havendo diferença estatística em relação aos demais períodos de jogo.

Conclusão Houve uma incidência de lesões musculares de 7,7 lesões $/ 1.000 \mathrm{~h}$, e ocorreram predominantemente nos jogos em casa, em defensores (laterais e zagueiros), com idade média de 28 anos, envolvendo principalmente a musculatura isquiotibial, com tempo médio de afastamento moderado (8 a 28 dias).
\end{abstract}

Trabalho desenvolvido no Centro de Traumatologia do Esporte, Escola Paulista de Medicina, Universidade Federal de São Paulo, São Paulo, Brasil. recebido

16 de Janeiro de 2020 aceito

10 de Março de 2020
DOI https://doi.org/

10.1055/s-0040-1712988. ISSN 0102-3616.
Copyright $\odot 2020$ by Sociedade Brasileira License terms de Ortopedia e Traumatologia. Published by Thieme Revinter Publicações Ltda, Rio de Janeiro, Brazil 


\begin{abstract}
Keywords

- athletic injuries

- athletes

- football

- hamstring muscles

Objective To perform a prospective evaluation of muscle injuries that occurred during the matches of series A and B of the Brazilian Men's Football Championship from 2016 to 2018.

Prospective A prospective-cohort study with data collection regarding muscle injuries that occurred during the official matches of the first and second divisions of the Brazilian Men's Soccer Championship in the 2016, 2017 and 2018 seasons.

Results The total number of muscle injuries was of 577 throughout the 3 seasons, with a gradual and annual reduction in the incidence of injuries (219 injuries in 2016, 195 in 2017, and 163 in 2018), with a statistically significant difference between the 2016 and 2018 seasons. Muscle injuries represented approximately 35\% of the total lesions. The incidence of muscle injuries was of 7.66 per 1,000 hours of play. During the 3 seasons (2016 to 2018), the most common injury was of the hamstring muscle ( $41.1 \%, 40.5 \%$, and $33.7 \%$ respectively). Wingers were the most affected players, and the most common injury severity scale was moderate ( 8 to 28 days). The moment of the match with the highest incidence of injuries was in the period between 61 and 75 minutes, with an index of $19.9 \%$, with no statistical difference in relation to the other periods of the match.

Conclusion There was an incidence of muscle injuries of $7.7 / 1,000 \mathrm{~h}$, and they occurred predominantly in home games, in defenders (wingers and centre-backs), with an average age of 28 years, mainly involving the hamstring muscles, with a moderate mean time of absence ( 8 to 28 days).
\end{abstract}

\section{Introdução}

O futebol é o esporte mais praticado no Brasil e no mundo. A prática profissional do futebol pode predispor o atleta ao desenvolvimento de lesões devido à maior exigência física, além de a uma maior exposição aos fatores de risco, como: número excessivo de jogos e treinamentos, alta demanda emocional, e presença de lesões prévias. ${ }^{1}$

Entre as lesões ocorridas em atletas profissionais de futebol, as musculares são as mais comuns, com uma incidência de aproximadamente $35 \%$ a $40 \% .^{2}$ Outro fator importante reside no fato de que, mesmo após a adoção de diversos programas de prevenção de lesões no futebol, como o da Federação Internacional de Futebol (Fédération Internationale de Football Association, FIFA, em francês), FIFA $11+$, Ekstrand et al., ${ }^{3}$ num estudo com acompanhamento de 11 anos, mostraram que, ao contrário das lesões ligamentares, não houve redução na incidência de lesões musculares neste período. Desta forma, as lesões musculares vêm sendo amplamente estudadas nos últimos anos devido ao alto impacto gerado sobre os clubes profissionais, tanto do ponto de vista esportivo quanto financeiro. ${ }^{4,5}$

No Brasil, apesar do aumento do interesse em avaliar as causas de afastamento e as principais lesões em jogadores profissionais de futebol, ainda se observa uma carência de trabalhos científicos nesta área, e, principalmente, de estudos centrados em lesões específicas deste esporte, como as musculares.

Portanto, o objetivo deste estudo é realizar uma avaliação prospectiva das lesões musculares ocorridas durante as partidas das séries A e B do Campeonato Brasileiro de Futebol Masculino dos anos de 2016 a 2018.

\section{Métodos}

Trata-se de um estudo de coorte prospectivo com coleta de dados referentes às lesões musculares ocorridas durante os jogos oficiais da primeira e segunda divisões do Campeonato Brasileiro de Futebol Masculino nas temporadas de 2016, 2017, e 2018. Os dados foram coletados por meio do portal médico online (portalmedico.cbf.com.br) da Confederação Brasileira de Futebol (CBF) ao final de cada temporada. Após o término das partidas, o preenchimento dos questionários de lesões foi realizado pelos médicos responsáveis de cada clube, devidamente registrados na CBF. Todos os atletas regularmente inscritos no Campeonato Brasileiro das séries A e B de 2016, 2017 e 2018, que participaram de pelo menos 1 jogo do torneio, foram incluídos no trabalho. Os atletas inscritos pelos clubes e que não atuaram em pelo menos um jogo durante o campeonato foram excluídos do estudo. 0 conceito usado para definir lesão foi o mesmo escolhido por Fuller et al. ${ }^{6}$ no consenso da FIFA de 2005: "Qualquer reclamação física sustentada por um jogador resultante de uma partida de futebol ou treinamento, independente da necessidade de atenção médica ou perda de tempo nas atividades de futebol".

O questionário preenchido pelos médicos continha diversas variáveis: posição de atuação no jogo, idade, número de partidas jogadas, e tempo de participação nos jogos. No que se refere às lesões, incluiu-se o registro de sua ocorrência, a 
localização, a descrição e gravidade da lesão, a recidiva, e o momento do campeonato no qual ocorreu a lesão.

As lesões foram classificadas de acordo com a gravidade, isto é, o tempo que o atleta ficou afastado até o retorno às atividades sem limitações: leves ( 1 a 3 dias), menores (4 a 7dias), moderadas ( 8 a 28 dias), maiores (28 dias a 8 semanas), e graves (mais de 8 semanas de afastamento). ${ }^{7}$

Após o retorno do atleta a jogos oficiais, o médico do clube preencheu outro questionário com dados referentes ao desfecho da lesão, como: tempo para retorno ao esporte, necessidade de intervenção cirúrgica, exames realizados, e severidade da lesão.

O risco de lesão foi calculado conforme consenso elaborado pela FIFA, em que a incidência de lesão é expressa pelo número de lesões por 1.000 horas de exposição (número de jogos $\mathrm{x}$ número de jogadores por partida $\mathrm{x}$ duração da partida em horas). ${ }^{6}$ Logo, a incidência lesões para cada 1.000 horas de jogo é calculada pela seguinte fórmula:

Incidência $=$ número total de lesões $\times 1.000$ horas / exposição.

O presente estudo foi aprovado pelo Comitê de Ética em Pesquisa em Seres Humanos sob o número 1.660.701, tendo os dados sido cedidos pela CBF.

\section{Análise Estatística}

As medidas de tendência central e de dispersão foram expressas em médias e desvios padrão(DPs) para as variáveis contínuas de distribuição simétrica, e em medianas e valores mínimo e máximo para as variáveis de distribuição assimétrica. As variáveis categóricas estão expressas em seus valores absolutos e relativos. A estimativa de diferença entre variáveis categóricas foi realizada pelos testes exato de Fisher e qui-quadrado de Pearson. Foi calculada a razão de chances (odds ratio, OR, em inglês) para quantificar o risco de lesões associadas a algumas variáveis de estudo.
Foram utilizados testes estatísticos não paramétricos, pois os dados eram quantitativos e contínuos. Utilizamos a análise de variância (analysis of variance, ANOVA, em inglês) para comparar as variáveis quantitativas. O teste de igualdade de duas proporções foi utilizado para comparar se a proporção de respostas de duas determinadas variáveis e/ou níveis eram estatisticamente significativos. Valores de $p<0,05$ foram considerados estatisticamente significativos. Todos os intervalos de confiança (IC) foram estabelecidos ao longo do trabalho com 95\% de confiança estatística.

\section{Resultados}

O Campeonato Brasileiro de Futebol contém 20 clubes em cada divisão, que se enfrentam entre em si ao longo de 38 rodadas por temporada. Em nosso estudo, foram coletados dados dos anos de 2016, 2017 e 2018, contabilizando 2.280 jogos. O número total de lesões musculares foi de 577 ao longo das 3 temporadas, havendo uma redução gradual e anual na incidência (219 lesões em 2016, 195 lesões em 2017, e 163 lesões em 2018), com diferença estatística significativa entre os anos de 2016 e 2018. Como houve um total de 1.663 lesões nas 3 temporadas, as musculares representaram aproximadamente $35 \%$ do total. A incidência das lesões musculares foi de 7,66 para cada 1.000 horas de jogo (-Figura 1). Nas 3 temporadas (2016 a 2018), a mais comum foi a lesão muscular dos isquiotibiais $(41,1 \%, 40,5 \%$, e $33,7 \%$, respectivamente).

Em relação à posição em campo dos atletas acometidos por lesões musculares, observamos uma maior prevalência de lesões nos: laterais (18,5\%), zagueiros (18,4\%), meiocampistas (16,6\%), atacantes (14,4\%), volantes (10,7\%), e goleiros (3,8\%) (-Figura 2). O grau de severidade mais comum foi o tipo moderado, com afastamento entre 8 e 28

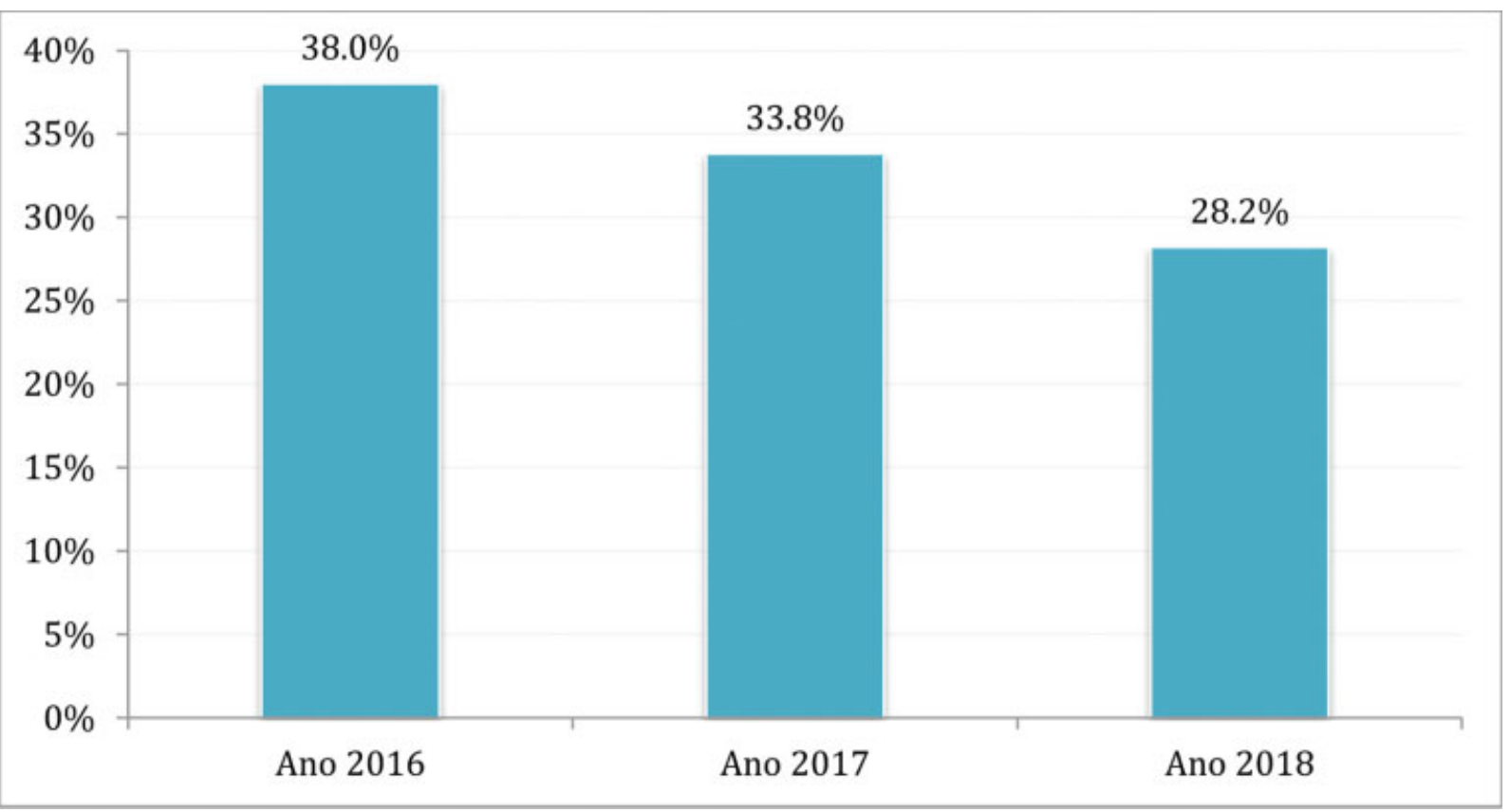

Fig. 1 Porcentagem de lesões musculares nas temporadas. 


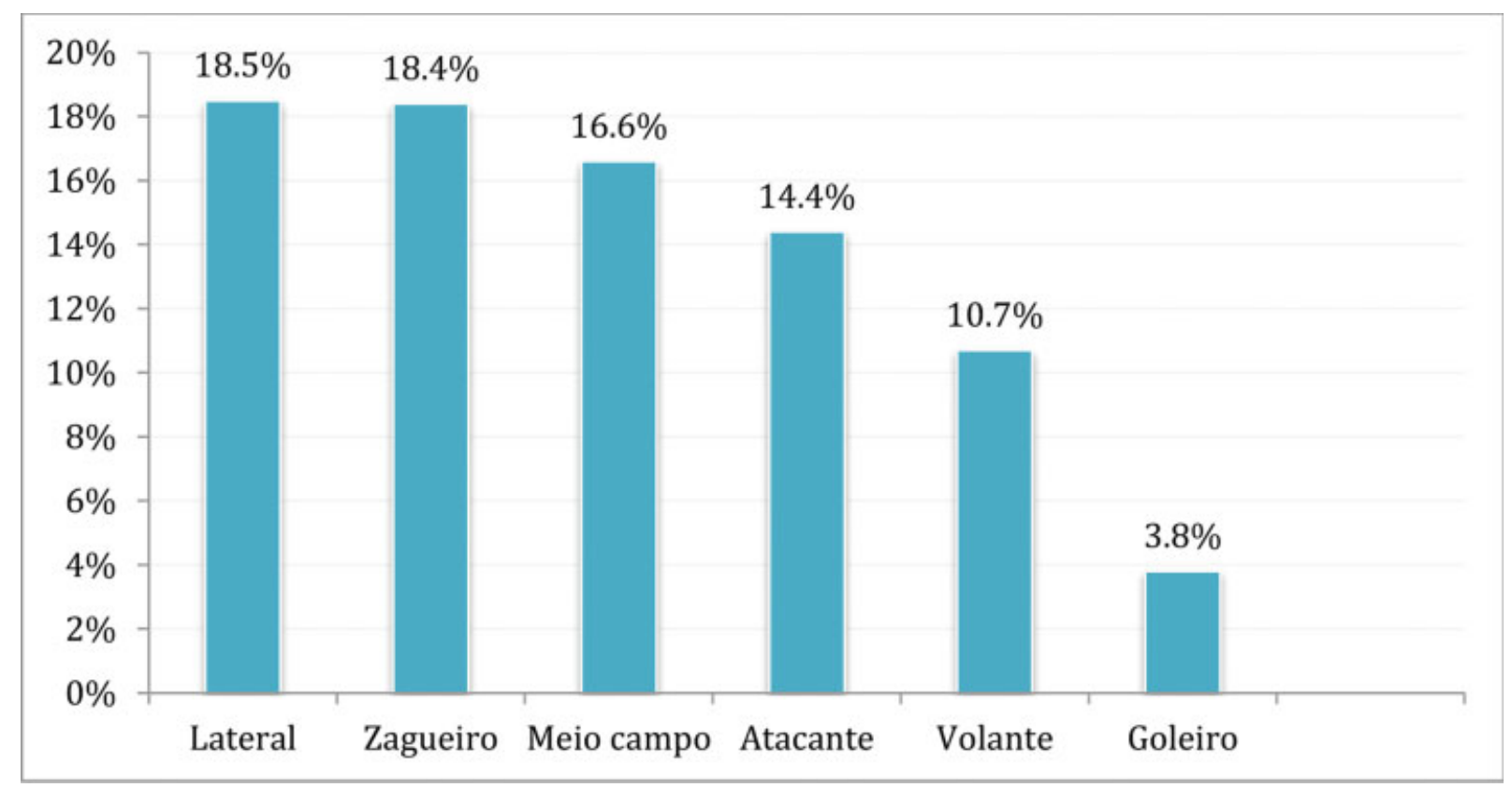

Fig. 2 Posição dos jogadores acometidos por lesões musculares.

dias, sendo a média de afastamento de $15,8 \pm 1,6$ dias. 0 grupo muscular mais acometido foi o dos músculos isquiotibiais. Os atletas acometidos por lesões musculares tinham uma média de aproximadamente 28 anos. Os clubes mandantes tiveram maior índice de lesão muscular (54,9\%), com significância estatística $(p<0,001)$. Com relação à localização (distância) da partida, houve maior prevalência de lesões musculares nos jogos em casa, e quando os clubes tiveram que percorrer distâncias maiores do que $800 \mathrm{~km}$ para o jogo (53,4\% e 33,4\%, respectivamente) (-Figura 3). A temperatura média nos jogos com lesões musculares foi de $22^{\circ} \mathrm{C}$. Sobre o clima, a maior parte das lesões musculares ocorreu com tempo ensolarado (38,6\%), mas não houve diferença estatística $(p=0,066)$ em relação às demais condições climáticas (-Figura 4).

O momento da partida com maior incidência de lesões $(19,9 \%)$ foi entre 61 e 75 minutos , não havendo diferença estatística em relação aos demais períodos de jogo ( - Figura 5). Ao longo das 3 temporadas, notamos que a média de lesões por clube foi caindo ao longo dos anos, pois em 2016 foram 5,92 lesões musculares por clube, contra 5,42 lesões em 2017, e 4,79 lesões em 2018.

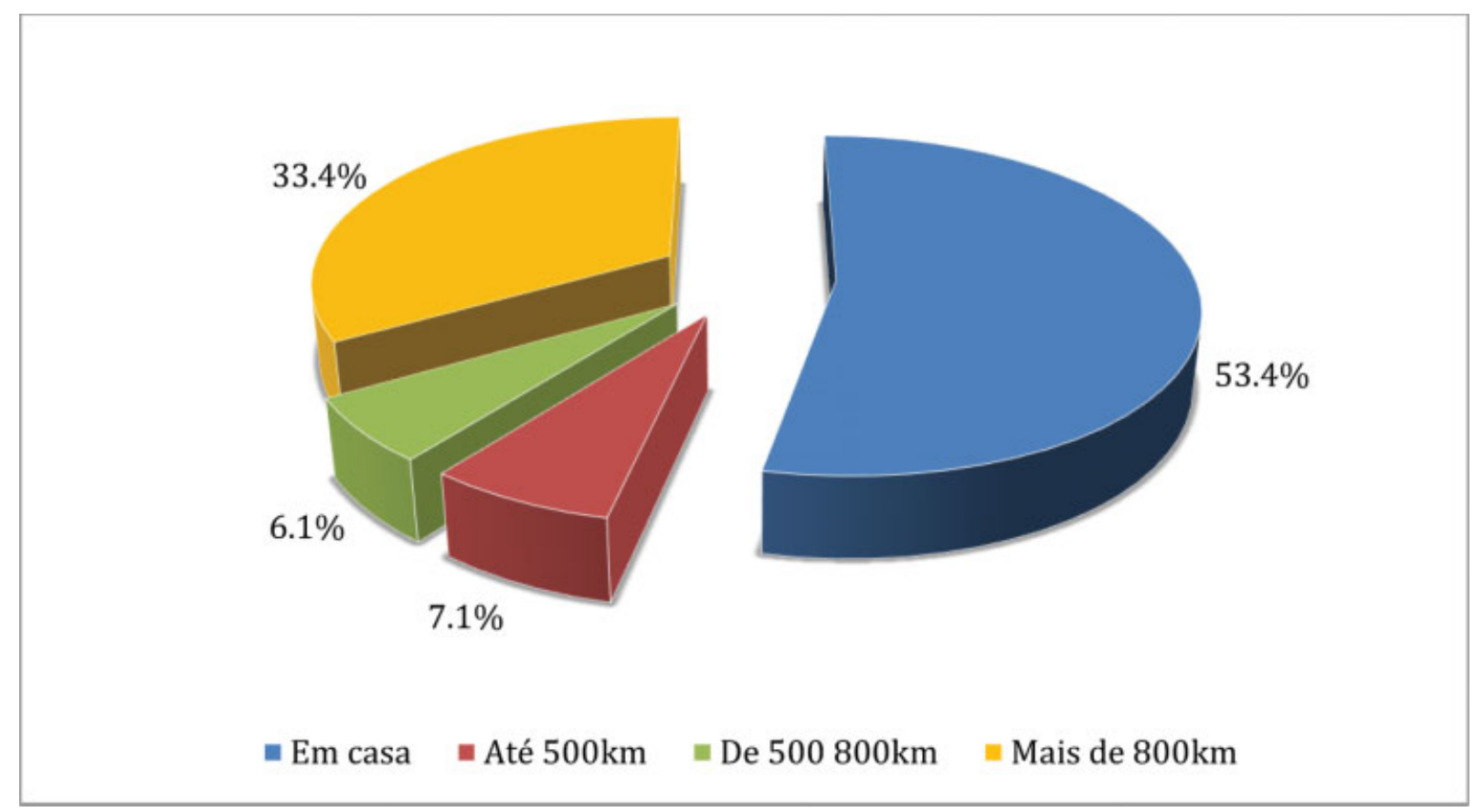

Fig. 3 Distribuição das doenças musculares de acordo com a distância da partida. 


\section{Distribuição de "Clima"}

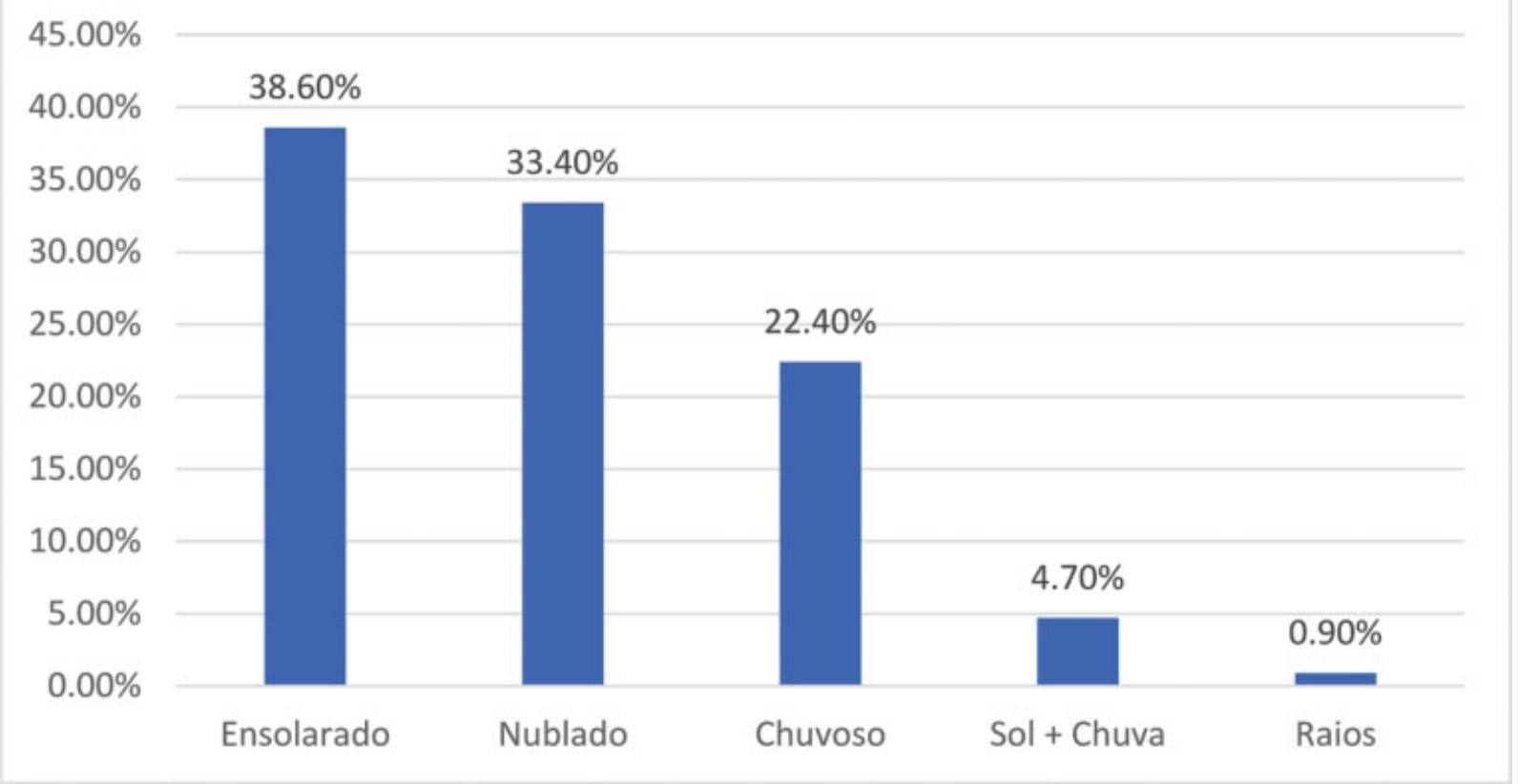

Fig. 4 Distribuição de lesões musculares de acordo com o clima da partida.

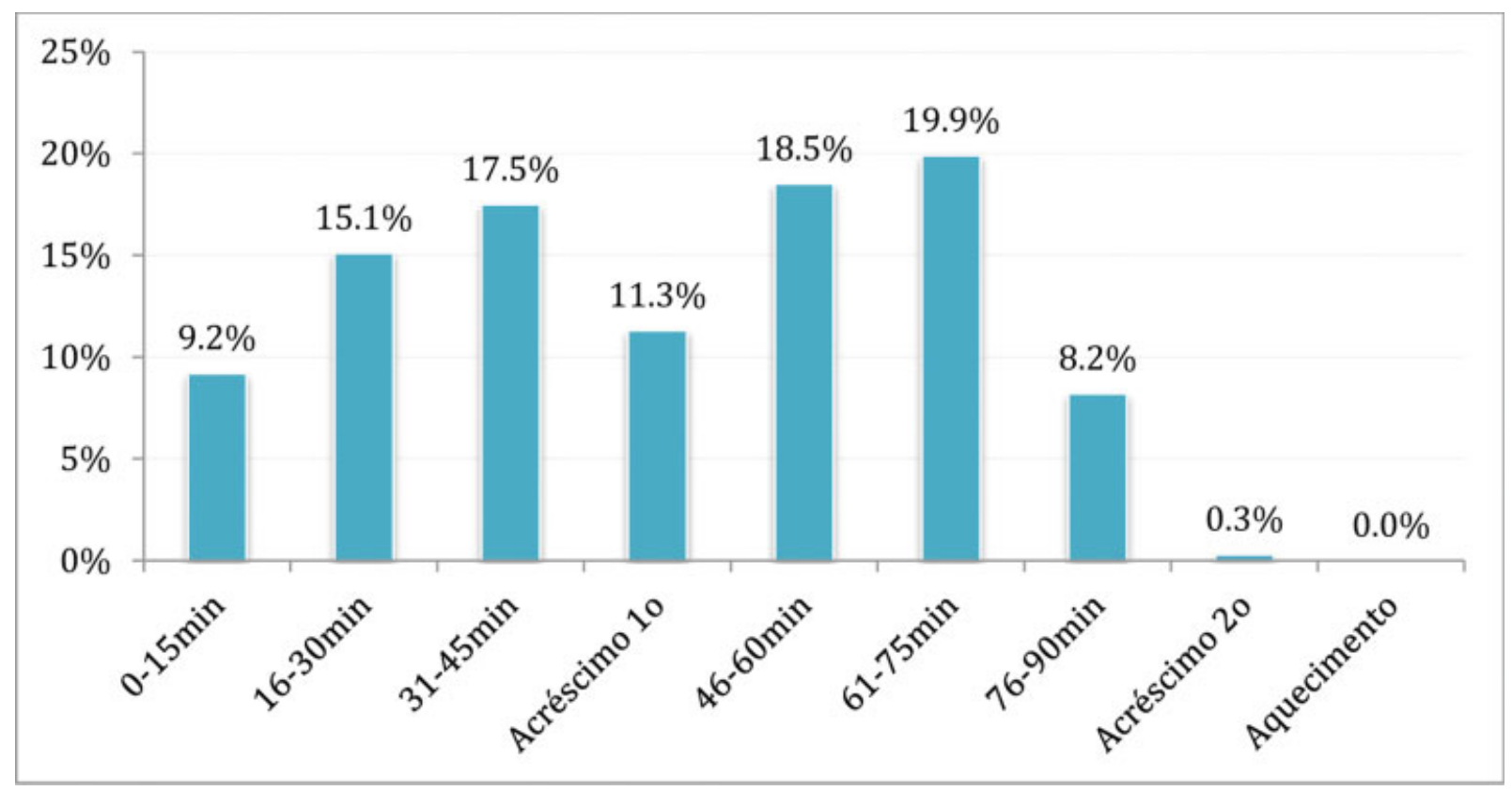

Fig. 5 Distribuição das lesões musculares de acordo com o momento da lesão.

Em relação aos turnos do campeonato, nos três anos avaliados, a maior parte das lesões musculares ocorreu no primeiro turno (-Figura 6).

\section{Discussão}

No Brasil, o futebol é o esporte mais praticado, e a paixão e o fanatismo dos torcedores por seus respectivos clubes transcendem a razão, sendo este um dos principais motivos da busca por melhores resultados e da obsessão por títulos.
Logo, a utilização máxima dos atletas é essencial aos clubes de futebol, que são prejudicados quando há lesão em seus atletas, inviabilizando-os à prática esportiva.

Em um estudo, Ekstrand et al. ${ }^{8}$ avaliaram 2.299 jogadores de clubes europeus entre 2001 e 2009 , e cerca de $1 / 3$ das lesões foram musculares, e as lesões do músculo isquiotibial foram a mais comuns, assim como no presente estudo.

Em outro estudo, Ekstrand et al. ${ }^{9}$ avaliaram os jogadores da Liga dos Campeões da União de Associações de Futebol Europeias (Union of European Football Associations, UEFA, 


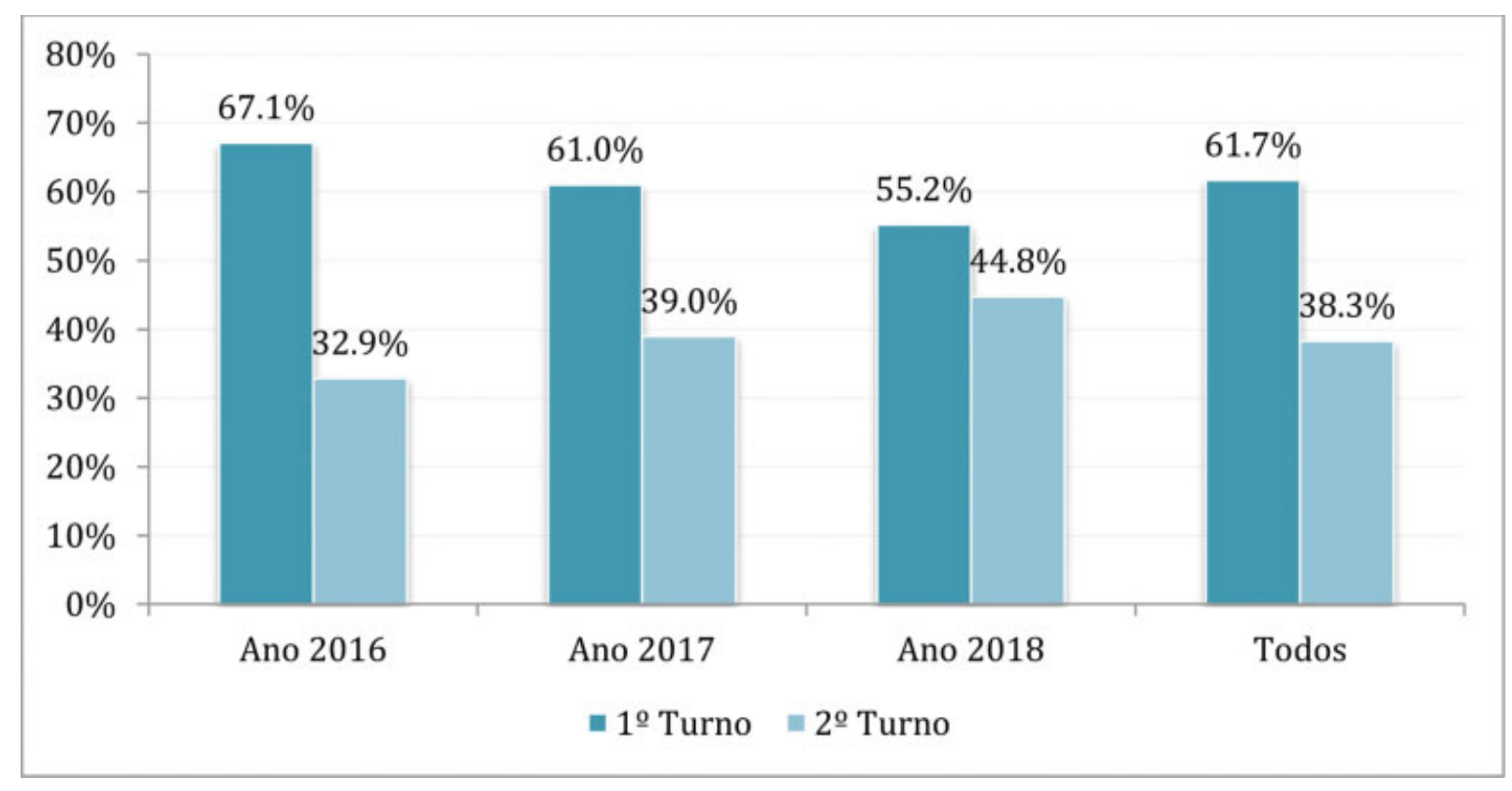

Fig. 6 Comparação de lesões musculares nos turnos do campeonato.

em inglês) durante 11 anos. Os autores observaram que houve diminuição da taxa de lesões ligamentares, mas não houve queda na taxa de lesões musculares. No presente estudo, houve uma queda progressiva nas três temporadas avaliadas. Estudos prévios ${ }^{2,10,11}$ que avaliaram o panorama de lesões em jogadores de futebol no Brasil demonstraram que as lesões musculares são as mais comuns, e uma hipótese que pode ser aventada é que, a partir desses estudos, houve uma maior preparação por parte dos clubes brasileiros para evitar lesões musculares, e isso justificaria essa queda nas três temporadas avaliadas no presente estudo. Outras hipóteses são aventadas, como melhora nas condições dos gramados no Brasil, clubes buscando elencos maiores, com mais jogadores, para preservar atletas, e o avanço no uso da tecnologia pelos departamentos médicos dos clubes.

Junge e Dvořák ${ }^{12}$ avaliaram as lesões ocorridas durante a Copa do Mundo FIFA de 2014 no Brasil; a mais comum foi a lesão muscular na coxa, e os autores recomendaram intervenções para prevenir lesões nos membros inferiores sem contato, as quais devem fazer parte da rotina de treinamento dos clubes de futebol.

Ekstrand et al. ${ }^{13}$ realizaram um acompanhamento durante 13 anos em clubes europeus de futebol, e observaram que houve um aumento na taxa de lesões musculares dos isquiotibiais durante os treinos e manutenção da taxa em relação aos jogos. Os autores colocaram como hipótese para essa diferença que há uma maior exigência muscular durante os treinamentos. No presente estudo, foram avaliadas apenas as lesões ocorridas durante os jogos, e houve uma diminuição na incidência de lesões musculares no decorrer das três temporadas.

No estudo de Bengtsson et al. ${ }^{14}$ em que foram avaliadas 14 temporadas consecutivas de clubes da UEFA, os autores encontraram uma média de 9,4 lesões musculares por 1.000 horas, valor um pouco acima do encontrado no presente estudo (7,66 lesões musculares per 1.000 horas). Outro aspecto abordado no estudo é que houve um número maior de lesões quando houve menos de três dias de descanso entre as partidas. Estudos prévios ${ }^{15-18}$ demonstraram redução de $20 \%$ no número de lesões quando houve mais de 3 dias de descanso entre as partidas. Sinais de fadiga muscular após uma partida de futebol duram cerca de 72 horas, o que foi demonstrado em estudos prévios. $^{19-22}$ Isso pode alterar os movimentos dos jogadores, levando os músculos a funcionar em níveis em que não estão acostumados, predispondo os atletas a novas lesões. Nas temporadas do Campeonato Brasileiro em que foram avaliadas as lesões, houve uma média de três dias de descanso entre as partidas, valor associado a um maior número de lesões. Além disso, no presente estudo, foi demonstrado que, em jogos fora de casa a uma distância maior do que $800 \mathrm{~km}$, houve uma incidência maior de lesões. Uma hipótese que pode ser aventada é a de que nessas partidas os jogadores tiveram menor tempo de descanso e maior desgaste devido à viagem, e, com isso, apresentaram um número maior de lesões.

No estudo de Buckthorpe et al., ${ }^{23}$ fez-se uma avaliação sobre as causas das lesões dos isquiotibiais, que são as mais comuns entre os jogadores de futebol. Os autores apontaram a importância do fortalecimento da musculatura do core e a integridade do balanço pélvico para evitar lesões dos isquiotibiais.

No estudo de Eirale, ${ }^{24}$ demonstrou-se que uma importante ferramenta para a diminuição da ocorrência de lesões dos isquiotibiais é a detecção de sintomas prodrômicos por parte dos atletas e/ou preparadores, e a realização de exames que detectam alterações antes do aparecimento da lesão, como a ressonância magnética e exames laboratoriais.

Ekstrand et al. ${ }^{25}$ avaliaram 35 times de futebol da Europa, e aqueles em que houve um período de parada no decorrer da temporada apresentaram um menor afastamento de jogadores por conta de lesões. Pode-se aventar a hipótese de que o menor índice de lesões em 2018 seja justificado pela parada devido ao período da Copa do Mundo. 
No estudo de Jones et al., ${ }^{26}$ foram avaliados 243 jogadores profissionais do futebol inglês, e demonstrou-se que as lesões musculares dos isquiotibiais são as mais frequentes, correspondendo a $39,5 \%$ de todas as lesões musculares e a $16,3 \%$ do total das lesões, taxa semelhante à encontrada no presente trabalho, assim como a escala de severidade da lesão moderada ( 8 a 28 dias), a qual foi a mais comum em ambos os estudos. No estudo de Klein et al., ${ }^{27}$ em que foram avaliadas 3 temporadas do campeonato alemão, a mais comum foi a lesão muscular da coxa, com $23,6 \%$ dos casos, e o grau de severidade mais comum foi o moderado ( 8 a 28 dias de afastamento).

Em relação ao turno em que ocorreram as lesões musculares, há necessidade de novos estudos que avaliem esse aspecto, pois no presente estudo a maioria delas ocorreu no primeiro turno, o que não é o mais esperado, uma vez que se sabe as lesões musculares estão muitas vezes associadas à fadiga muscular, a qual é mais esperada no segundo turno.

As lesões musculares, umas das principais causas de afastamento dos atletas do âmbito esportivo, vêm sendo amplamente estudadas para a compreensão dos fatores de risco e o estabelecimento do tratamento adequado. Com esse entendimento, haverá ganho esportivo, seja dos clubes, dos jogadores, ou do campeonato, que terá seu nível de qualidade aumentado, e os próprios torcedores também vão se beneficiar, pois verão em campo seus ídolos, aumentando o ganho financeiro e esportivo dos envolvidos.

No Brasil, apesar do alto valor financeiro envolvido nas duas principais divisões do Campeonato Brasileiro de Futebol, nota-se uma carência de estudos sobre as causas de impedimento à prática esportiva de seus atletas, entre elas as lesões musculares.

Uma limitação do presente estudo é a possibilidade de viés de informação, posto que as informações podem ter sido modificadas ou até omitidas pelos médicos dos clubes. Além disso, o estudo avaliou apenas lesões agudas ocorridas durante os jogos, e não avaliou as lesões sofridas durante o treinamento e as doenças não relacionadas ao esporte. Outra limitação é que o tempo de exposição foi calculado baseado em 22 jogadores e 90 minutos por jogo. Um método mais preciso seria considerar os acréscimos ou a duração real de cada partida e o número de minutos de exposição para cada jogador individualmente. Entre os pontos fortes, o presente estudo é o primeiro no Brasil a realizar a avaliação de lesões musculares por três anos no principal campeonato de futebol do país. Além disso, foram realizadas correlações que podem ser conduzidas em outros estudos para comparação, e este estudo confirmou certas associações que, apesar de esperadas, não tinham sido previamente avaliadas.

\section{Conclusão}

As lesões musculares em jogadores de futebol profissional das séries A e B do Campeonato Brasileiro de Futebol entre os anos 2016 a 2018 corresponderam a 35\% das lesões, com uma incidência de 7,7 /1,000 h, e ocorreram predominantemente nos jogos em casa, em defensores (laterais e zagueiros), com idade média de 28 anos, envolvendo principalmente a mus- culatura isquiotibial, com tempo médio de afastamento moderado (8 a 28 dias).

Apesar do avanço nos estudos epidemiológicos e demográficos das lesões no futebol, concluímos que a literatura carece de novos estudos, e que há margem para novas pesquisas sobre esse tema.

\section{Conflito de Interesses}

Os autores declaram não haver conflito de interesses.

Agradecimentos

Gostaríamos de agradecer os médicos dos clubes e a Confederação Brasileira de Futebol (CBF) pela ajuda para a realização desse trabalho.

\section{Referências}

1 Arliani GG, Lara PHS, Astur DC, Pedrinelli A, Pagura JR, Cohen M. Orthopaedics injuries in male professional football players in Brazil: a prospective comparison between two divisions. Muscles Ligaments Tendons J 2018;7(03):524-531

2 Netto DC, Arliani GG, Thiele ES, Cat MNL, Cohen M, Pagura JR. Prospective Evaluation of Injuries occurred during the Brazilian Soccer Championship in 2016. Rev Bras Ortop (Sao Paulo) 2019; 54(03):329-334

3 Ekstrand J, Hägglund M, Kristenson K, Magnusson H, Waldén M. Fewer ligament injuries but no preventive effect on muscle injuries and severe injuries: an 11-year follow-up of the UEFA Champions League injury study. Br J Sports Med 2013;47(12):732-737

4 Eirale C, Tol JL, Farooq A, Smiley F, Chalabi H. Low injury rate strongly correlates with team success in Qatari professional football. Br J Sports Med 2013;47(12):807-808

5 Hägglund M, Waldén M, Magnusson H, Kristenson K, Bengtsson H, Ekstrand J. Injuries affect team performance negatively in professional football: an 11-year follow-up of the UEFA Champions League injury study. Br J Sports Med 2013;47(12):738-742

6 Fuller CW, Ekstrand J, Junge A, et al. Consensus statement on injury definitions and data collection procedures in studies of football (soccer) injuries. Br J Sports Med 2006;40(03):193-201

7 Arliani GG, Belangero PS, Runco JL, Cohen M. The Brazilian Football Association (CBF) model for epidemiological studies on professional soccer player injuries. Clinics (São Paulo) 2011;66 (10):1707-1712

8 Ekstrand J, Hägglund M, Waldén M. Epidemiology of muscle injuries in professional football (soccer). Am J Sports Med 2011; 39(06):1226-1232

9 Ekstrand J, Hägglund M, Waldén M. Injury incidence and injury patterns in professional football: the UEFA injury study. Br J Sports Med 2011;45(07):553-558

10 Arliani GG, Lara PHS, Astur DC, Pedrinelli A, Pagura JR, Cohen M. Prospective evaluation of injuries occurred during a professional soccer Championship in 2016 in São Paulo, Brazil. Acta Ortop Bras 2017;25(05):212-215

11 de Moraes ER, Arliani GG, Lara PHS, da Silva EHR, Pagura JR, Cohen M. Orthopedic injuries in men's professional soccer in Brazil: prospective comparison of two consecutive seasons 2017/2016. Acta Ortop Bras 2018;26(05):338-341

12 Junge A, Dvořák J. Football injuries during the 2014 FIFA World Cup. Br J Sports Med 2015;49(09):599-602

13 Ekstrand J, Waldén M, Hägglund M. Hamstring injuries have increased by $4 \%$ annually in men's professional football, since 2001: a 13-year longitudinal analysis of the UEFA Elite Club injury study. Br J Sports Med 2016;50(12):731-737

14 Bengtsson H, Ekstrand J, Waldén M, Hägglund M. Muscle injury rate in professional football is higher in matches played within 5 days since the previous match: a 14-year prospective study with 
694 Estudo prospectivo das lesões musculares em jogos do Campeonato Brasileiro Margato et al.

more than 130000 match observations. Br J Sports Med 2018;52 (17):1116-1122

15 Bengtsson H, Ekstrand J, Waldén M, Hägglund M. Match injury rates in professional soccer vary with match result, match venue, and type of competition. Am J Sports Med 2013;41(07): 1505-1510

16 Dupont G, Nedelec M, McCall A, McCormack D, Berthoin S, Wisløff U. Effect of 2 soccer matches in a week on physical performance and injury rate. Am J Sports Med 2010;38(09):1752-1758

17 Della Villa F, Mandelbaum BR, Lemak LJ. The Effect of Playing Position on Injury Risk in Male Soccer Players: Systematic Review of the Literature and Risk Considerations for Each Playing Position. Am J Orthop 2018;47(10):. Doi: 10.12788/ ajo.2018.0092

18 Carling C, McCall A, Le Gall F, Dupont G. The impact of short periods of match congestion on injury risk and patterns in an elite football club. Br J Sports Med 2016;50(12):764-768

19 Ascensão A, Rebelo A, Oliveira E, Marques F, Pereira L, Magalhães J. Biochemical impact of a soccer match - analysis of oxidative stress and muscle damage markers throughout recovery. Clin Biochem 2008;41(10-11):841-851

20 Fatouros IG, Chatzinikolaou A, Douroudos II, et al. Time-course of changes in oxidative stress and antioxidant status responses following a soccer game. J Strength Cond Res 2010;24(12): 3278-3286
21 Mohr M, Draganidis D, Chatzinikolaou A, et al. Muscle damage, inflammatory, immune and performance responses to three football games in 1 week in competitive male players. Eur J Appl Physiol 2016;116(01):179-193

22 Nedelec M, McCall A, Carling C, Legall F, Berthoin S, Dupont G. The influence of soccer playing actions on the recovery kinetics after a soccer match. J Strength Cond Res 2014;28(06):1517-1523

23 Buckthorpe M, Gimpel M, Wright S, Sturdy T, Stride M. Hamstring muscle injuries in elite football: translating research into practice. Br J Sports Med 2018;52(10):628-629

24 Eirale C. Hamstring injuries are increasing in men's professional football: every cloud has a silver lining? Br J Sports Med 2018;52 (23): 1489

25 Ekstrand J, Spreco A, Davison M. Elite football teams that do not have a winter break lose on average 303 player-days more per season to injuries than those teams that do: a comparison among 35 professional European teams. Br J Sports Med 2019;53(19):1231-1235

26 Jones RN, Greig M, Mawéné Y, Barrow J, Page RM. The influence of short-term fixture congestion on position specific match running performance and external loading patterns in English professional soccer. J Sports Sci 2019;37(12):1338-1346

27 Klein C, Luig P, Henke T, Platen P. Injury burden differs considerably between single teams from German professional male football (soccer): surveillance of three consecutive seasons. Knee Surg Sports Traumatol Arthrosc 2020;28(05):1656-1664 